\title{
Triangular resection versus folding repair for simple posterior mitral leaflet lesions: case-control study
}

\author{
Ken Nakamura ${ }^{1}$, Kouan Orii ${ }^{1}$, Takayuki Abe ${ }^{1}$, Hirofumi Haida ${ }^{1}$, Kazuhiro Hashimoto ${ }^{2}$, Takashi Kunihara $^{2}$ \\ ${ }^{1}$ Department of Cardiac Surgery, Saitama Cardiovascular and Respiratory Center, Kumagaya-shi, Saitama, Japan; ${ }^{2}$ Department of Cardiac Surgery, \\ Jikei University School of Medicine, Minato-Ku, Tokyo, Japan \\ Contributions: (I) Conception and design: K Nakamura, K Hashimoto; (II) Administrative support: T Abe, H Haida; (III) Provision of study materials \\ or patients: K Nakamura, K Orii; (IV) Collection and assembly of data: K Nakamura; (V) Data analysis and interpretation: K Nakamura, T \\ Kunuhara; (VI) Manuscript writing: All authors; (VII) Final approval of manuscript: All authors. \\ Correspondence to: Ken Nakamura, MD, PhD. Department of Cardiac Surgery, Saitama Cardiovascular and Respiratory Center, 1696, Itai, Kumagaya- \\ shi, Saitama, 360-0197, Japan. Email: cardiacsurgken@ybb.ne.jp; kenkentakota@yahoo.co.jp.
}

Background: A triangular resection (TR)/suture of the posterior leaflet lesion is the most common technique in mitral valve repair procedures. However, posterior leaflet motion is restricted after surgical resection in echocardiogram analyses. Although several reports have compared the resection/suture technique and the artificial chorda technique, few reports have compared TR with folding repair (FR). We compared the effectiveness and short-term impact of the TR and non-resection-based FR procedures on patients undergoing mitral valve repair.

Methods: Mitral valve repair was conducted on 36 patients with moderate to severe mitral regurgitation (MR) through either TR $(n=18)$ or FR $(n=18)$. Echocardiographic data were collected pre- and postoperatively. Routine echocardiographic follow-ups were performed for each patient. Data were analyzed using t-test, Mann-Whitney U-test, chi-squared analysis, or Fisher's exact test. $\mathrm{P}$ values $<0.05$ were considered significant.

Results: Operative time and length of stay within the intensive care unit and hospital did not significantly differ between the two techniques. Post-operative echocardiographic results showed a significantly larger mitral valve area after FR $(\mathrm{P}=0.029)$. The regurgitation regression rate was $94 \%$ under mild $\mathrm{MR}$ in the FR group ( 1 case; severe) and $88.8 \%$ in the TR group ( 2 cases; moderate) during the same duration.

Conclusions: Both techniques showed comparable outcomes and durability. However, FR is relatively simple and it is possible to re-initiate FR procedures; thus, it may also provide an opportunity for lessexperienced surgeons to conduct valve repair surgeries. The study was registered with the University Hospital Medical Information Network (study ID: UMIN000039041).

Keywords: Triangular resection (TR); folding repair (FR); mitral valve repair

Submitted Sep 02, 2020. Accepted for publication Nov 16, 2020.

doi: $10.21037 / \mathrm{cdt}-20-760$

View this article at: http://dx.doi.org/10.21037/cdt-20-760

\section{Introduction}

The fundamental approach used for successful mitral valve repair is based on Carpentier's techniques and includes (I) resection and suturing, (II) chordae reconstruction, (III) edge-to-edge repair, (IV) patch augmentation repair, and (V) ring annuloplasty (1). Combinations of these techniques have previously been used for mitral regurgitation (MR) treatment. The triangular or quadrangular resection suture procedures, which focuses on the posterior leaflet lesion of the mitral valve, are the commonly utilized approaches. However, echocardiographic analyses conducted after these resection suture procedures have indicated that these techniques may restrict the motion of the posterior 
leaflet $(2,3)$. Perier et al. introduced a new paradigm for the reconstruction of a posterior leaflet prolapse, which they termed "respect rather than resect (RRR)". The goal of the RRR approach is to correct the prolapse, without leaflet resection, and to transform the posterior leaflet into a smooth and vertical buttress to ensure the best coaptation surface (4).

The resection suture technique remains the main technique for the surgical reconstruction of the heart valve. However, incorporation of a non-resection-based folding technique, which can utilize artificial chordae, allows for simplified procedure reversal and repositioning. Currently, our institution has begun to actively incorporate techniques, such as the folding or non-resection technique using artificial chorda, because of their reversibility and retry on repair, aiming to shift to minimally invasive cardiac surgery (MICS).

Although several reports have compared the resection/ suture technique and the artificial chorda technique (3,5-7), few reports have compared triangular resection (TR) with folding repair (FR). In this study, we compared the effectiveness and short-term impact of the TR technique and the non-resection-based FR procedure on patients undergoing mitral valve repair.

We present the following article in accordance with the STROBE reporting checklist (available at http://dx.doi. org/10.21037/cdt-20-760).

\section{Methods}

\section{Study design and subjects}

This study conformed to the provisions of the Declaration of Helsinki (as revised in 2013). This study was approved by the institutional review board at the Saitama Cardiovascular and Respiratory Center (No.2020004) as a Case Control Study. Written informed consent was obtained from the patient for using the data for academic publication purposes. The study was registered with the University Hospital Medical Information Network (study ID: UMIN000039041).

Between January 2016 and September 2019, a total of 88 consecutive patients with moderate-to-severe MR underwent mitral valve repair at the Saitama Cardiovascular and Respiratory Center. Only patients who underwent mitral valve repair for the posterior mitral leaflet (PML) lesion were included in this study. Patients who (I) underwent a concomitant aortic valve procedure, (II) had anterior leaflet or bi-leaflet pathology, or (III) required reoperation were excluded. To mitigate any confounding factors, patients with neochordal replacement were further excluded. A total of 36 patients, who were treated with either triangular suture resection (TR; $n=18)$ or FR $(n=18)$, were assessed in this study.

\section{Operative techniques (Figure S1)}

FR is based on a nonresectional technique with vertical inversion of the prolapsed segment into the left ventricle. The prolapsed lesion was detected by a saline water pressure test and indicated by a markedly redundant tissue. A pilot suture was placed at the free edge of the excessive lesion. A temporary suture was placed, followed by an additional saline water test. If the saline pressure test showed regression of $M R$, additional interrupted sutures were placed toward the annulus. The leaflet height was adjusted to prevent the formation of an indentation, which can lead to a new MR lesion. A ring selection was based on the measurement of the surface area of the anterior leaflet and placed after confirming the absence of MR to prevent future annulare dilation. TR was performed by resecting excessive leaflet tissue, then reattaching leaflet remnants, in the absence of an annular plication (8). To obtain a good operative field, the ring annuloplasty sutures were placed first, regardless of the procedure (FR or TR). The choice of FR or TR was based on the surgeon's preference. These procedures were performed as basic techniques by three surgeons.

\section{Echocardiographic assessment and follow-up}

To acquire hemodynamic and geometric data before the mitral valve repair, transesophageal echocardiography and transthoracic echocardiography (TTE) were performed by two experienced echocardiographers using a Philips EPIQ CVx machine (Phillips HealthCare, Hamburg Germany). Conventional echocardiographic data, including left ventricular (LV) diastolic and systolic dimension (Dd, Ds), $\mathrm{LV}$ ejection fraction (EF), left atrial diameter, the grade of MR, and mean mitral valve pressure gradient (MVPG), were collected. We also collected data on the levels of brain natriuretic peptide (BNP) pre- and post-operatively. Routine follow-up evaluations with TTE were performed between 7 and 37 months (17.74 \pm 8.31 months). 
Table 1 Clinical profiles of the patients in each group

\begin{tabular}{lccc}
\hline Characteristic & TR & FR & P value \\
\hline No. of patients & 18 & 18 & \\
Sex: male & 12 & 12 & \\
Age (mean \pm SD) & $69.7 \pm 10$ & $66.3 \pm 7.7$ & 0.286 \\
NYHA class (median) & 1 & 3 & $<0.001$ \\
I-II N [\%] & $14[78]$ & $9[50]$ & \\
III-IV N [\%] & $4[22]$ & $9[50]$ & \\
BNP (pg/mL) & $167 \pm 245$ & $237 \pm 438$ & \\
Complication N [\%] & & & \\
COPD & $2[11]$ & $1[5.5]$ & 0.546 \\
Af & $2[11]$ & $2[11]$ & 0.590 \\
CKD & $1[5.5]$ & $2[11]$ & 0.546 \\
DM & $3[16]$ & $2[11]$ & 0.629 \\
IE (healed) & $4[22]$ & $1[5.5]$ & 0.148 \\
\hline
\end{tabular}

Values are mean $\pm S D$ or percentages in parentheses. TR, triangular resection; FR, folding repair; $\mathrm{BNP}$, brain natriuretic peptide; COPD, chronic obstructive pulmonary disease; CKD, chronic kidney disease; DM, diabetes mellitus; IE, infective endocarditis; SD, standard deviation.

Table 2 Pre-operative echocardiographic assessment variables

\begin{tabular}{lccc}
\hline Characteristic & TR & FR & P value \\
\hline LVEF $(\%)$ & $66.4 \pm 7.3$ & $64.0 \pm 11.0$ & 0.49 \\
LVESd $(\mathrm{mm})$ & $33.0 \pm 5.5$ & $37.0 \pm 7.1$ & 0.11 \\
LVEDd $(\mathrm{mm})$ & $54.0 \pm 5.5$ & $58.0 \pm 6.7$ & 0.13 \\
LAD $(\mathrm{mm})$ & $48.5 \pm 5.8$ & $50.0 \pm 7.7$ & 0.36 \\
MR degree & Severe 18 & Severe 17, & \\
& & moderate 1 & \\
Location of & & & \\
prolapse $\mathrm{n}(\%)$ & & & \\
P1 & $1(5.5)$ & $5(27.7)$ & \\
P2 & $14(77.7)$ & $3(16.6)$ & \\
P3 & $3(16.6)$ & $10(55.5)$ & \\
Chordal rupture & $11(61.1)$ & $7(38.8)$ & \\
With commissure & 0 & $7(38.8)$ & \\
or complex lesion & &
\end{tabular}

TR, triangular resection; FR, folding repair; LAD, left atrial diameter; LVEF, left ventricular ejection fraction.

\section{Statistical analysis}

Operative data were retrospectively analyzed using StatMate V (ATMS Inc., Tokyo, Japan) and IBM SPSS Statistics for Windows (version 26.0; IBM Corp., Armonk, NY, USA). Continuous variables are presented as means \pm standard deviations or medians with range and were compared using the Student's $t$-test or the Mann-Whitney $U$-test. Categorical variables were analyzed by the $\chi^{2}$ test or the Fisher's exact test. A probability value of $<0.05$ was considered significant.

\section{Results}

Between January 2016 and September 2019, 36 patients received mitral valve repair for a PML (TR $n=18 v s$. FR $\mathrm{n}=18$ ). The baseline preoperative characteristics of both groups were shown in Table 1 . The mean age was $69.7 \pm$ 10 years in the TR group and $66.3 \pm 7.7$ years in the FR group $(\mathrm{P}=0.286)$. According to the functional classifications by the New York Heart Association (NYHA), members of the FR group had more severe levels of heart failure (median: TR 1.0 vs. FR 3.0, $\mathrm{P}<0.001$ ). A large percentage of patients in the TR group exhibited complicated infective endocarditis (IE) ( $\mathrm{TR} \mathrm{n}=4,22.2 \%$ vs. $\mathrm{FR} \mathrm{n}=1,5.5 \%$; $\mathrm{P}=0.148)$.

Preoperative echocardiographic data of the two groups (TR vs. FR) are shown in Table 2. According to the preoperative echocardiographic results, cardiac function and morphology showed no statistically significant differences between the two groups. However, the location of prolapse was $77.7 \% \mathrm{P} 2$ in TR and $55.5 \% \mathrm{P} 3$ in FR (TR-P2 $\mathrm{n}=14$; FR-P1, 3 n=17).

The operative time involved in cardiopulmonary bypass (CPB) was comparable between the two groups. Furthermore, the length of stay in the intensive care unit (ICU) and in the hospital was not statistically significant between the two groups. However, the ring size was significantly larger in the FR group $(\mathrm{P}=0.024)$ (Table 3).

The levels of brain natriuretic peptide (BNP) decreased significantly from $167 \pm 245 \mathrm{pg} / \mathrm{mL}$ pre-operatively to $69 \pm 9.8 \mathrm{pg} / \mathrm{mL}$ post-operatively $(\mathrm{P}=0.012)$ in the TR group. However, there was no significant change in the respective BNP levels in the FR group $(237 \pm 438$ to $57 \pm 39$; $\mathrm{P}=0.081)$. There was no statistically significant difference between the TR and FR groups in the BNP values after the operation 
Table 3 Baseline characteristics of the two groups peri-operatively

\begin{tabular}{lccc}
\hline Characteristic & TR & FR & P value \\
\hline Operation time (min) & $280 \pm 59$ & $265 \pm 45$ & 0.48 \\
CPB (min) & $173 \pm 43$ & $171 \pm 35$ & 0.88 \\
AoCT (min) & $125 \pm 32$ & $125 \pm 30$ & 0.95 \\
Ring size (mm) & 29.5 & 30.7 & 0.024 \\
Extubation time (hour) & $13.5 \pm 7.3$ & $10.5 \pm 6.1$ & 0.25 \\
ICU stay (days) & $4.0 \pm 1.3$ & $3.8 \pm 1.6$ & 0.77 \\
Hospital stay (days) & $20 \pm 6.2$ & $18 \pm 4.0$ & 0.31 \\
\hline
\end{tabular}

The baseline characteristics of both two group at a perioperative state. TR, triangular resection; FR, folding repair; AoCT, aorta clamping time; CPB, cardiopulmonary bypass; ICU, intensive care unit.

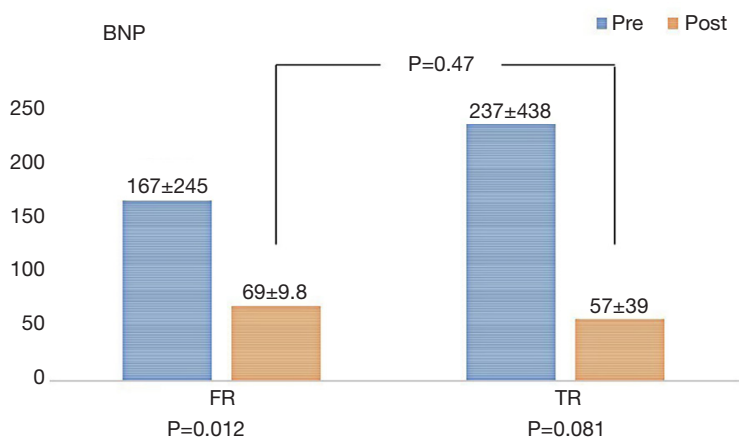

Figure 1 The brain natriuretic peptide (BNP) level changes postoperatively compared to the pre-operative levels. There was a significant decrease observed in the triangular suture resection (TR) group.

$(\mathrm{P}=0.47)$ (Figure 1).

\section{Echocardiographic data}

A comparison of the post-operative echocardiographic data between the TR and FR

There were no statistically significant differences in EF (Figure 2; $\mathrm{P}=0.08)$, $\mathrm{Dd}(\mathrm{P}=0.25)$, $\mathrm{Ds}(\mathrm{P}=0.08)$, and $\mathrm{LAD}$ $(\mathrm{P}=0.46)$ between the two groups (Table 4). The mitral valve area (MVA) was significantly larger in the FR group than in the TR group $\left(2.5 \pm 0.5\right.$ vs. $\left.3.16 \pm 0.9 \mathrm{~cm}^{2} ; \mathrm{P}=0.029\right)$. However, the mean diastolic MV gradient in the TR $(3.8 \pm 0.4 \mathrm{mmHg})$ and FR $(2.8 \pm 0.4 \mathrm{mmHg})$ groups was not statistically significant $(\mathrm{P}=0.09)$ (Table 4).
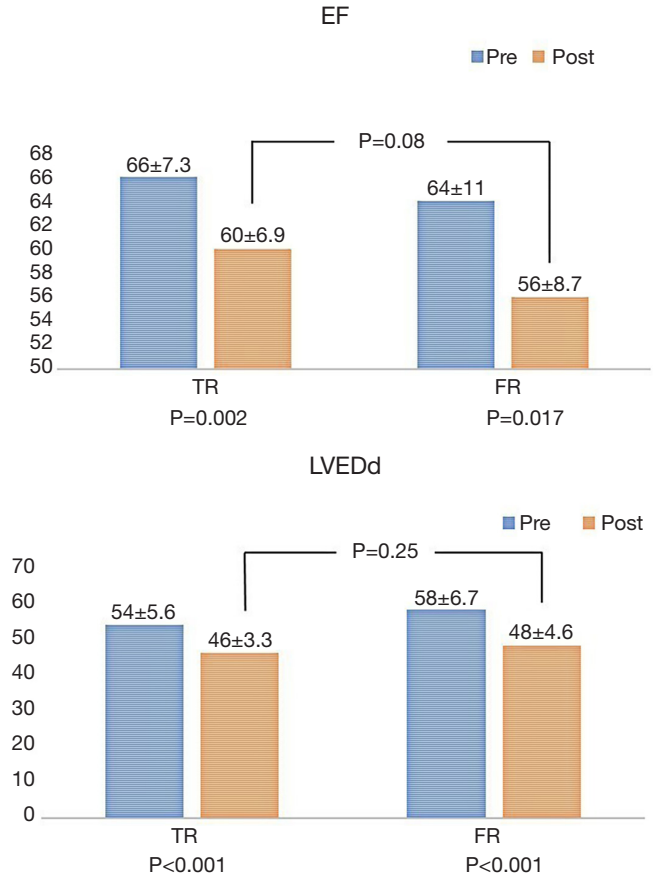

Figure 2 Comparison of the EF and LVEDd obtained preoperatively with those obtained post-operatively. There were no statistically significant differences in ejection fraction $(\mathrm{EF})(\mathrm{P}=0.08)$ and diastolic dimension (Dd) $(\mathrm{P}=0.25)$. The EF decreased in both groups.

\section{A comparison of TR and FR through pre- and post- operative echocardiographic data analysis}

After the operation, the EF value decreased to $60 \% \pm 6.9 \%$ from a pre-operative value of $66 \% \pm 7.3 \%(\mathrm{P}=0.02)$ in the TR group, whereas in the FR group, EF decreased to $56 \% \pm 8.7 \%$ from $64 \% \pm 11 \%(\mathrm{P}=0.017)$ (Tables 2,4$)$. The LVEDd values decreased to $46 \pm 3.3 \mathrm{~mm}$, from a preoperative value of $54 \pm 5.6 \mathrm{~mm}(\mathrm{P}<0.001)$ in the TR group. Similarly, the FR group displayed a decrease in LVEDd values from $58 \pm 67$ to $48 \pm 4.6 \mathrm{~mm}(\mathrm{P}<0.001)$ (Tables 2,4; Figure 3). However, there was no difference between the two groups, postoperatively.

The MR regression rate was $94 \%$ under mild MR in the FR group ( 1 case; severe MR), while it was $88.8 \%$ in the TR group ( 2 cases; moderate MR) during the same duration (Figures 3,4). The reasons for recurrence were a degenerative stenotic change [mitral stenosis (MS) $n=2$ ] in the TR group and a chordae rupture in the FR group $(n=1)$. There were no re-operation cases during the observation period in either group. 


\section{Discussion}

PML prolapse is the most common lesion in mitral valve insufficiency. The TR technique, a standard technique for mitral lesion repair, has exhibited excellent long-term durability (9). However, Perier et al. suggested that repair procedures should preserve the posterior leaflet tissue to maintain anatomical and physiological distributions of forces and stress on valve components in the left

Table 4 Comparison of the post-operative echocardiographic data between the TR and FR

\begin{tabular}{lccc}
\hline Characteristic & TR & FR & P value \\
\hline LVEF $(\%)$ & $60.5 \pm 4.3$ & $56.0 \pm 8.7$ & 0.08 \\
LVEDd $(\mathrm{mm})$ & $46.4 \pm 3.3$ & $48.0 \pm 4.6$ & 0.25 \\
LVESd $(\mathrm{mm})$ & $31.4 \pm 2.1$ & $33.0 \pm 5.1$ & 0.08 \\
LAD $(\mathrm{mm})$ & $42.5 \pm 5.4$ & $44.5 \pm 8.7$ & 0.46 \\
MVA $\left(\mathrm{cm}^{2}\right)$ & $2.5 \pm 0.5$ & $3.16 \pm 0.9$ & 0.029 \\
MVPG $(\mathrm{mmHg})$ & $3.8 \pm 0.4$ & $2.8 \pm 0.4$ & 0.09 \\
MR degree & & & \\
None & 2 & 1 & \\
Trivial & 6 & 3 & \\
Mild & 8 & 0 & \\
Moderate & 2 & 1 & \\
Severe & 0 & & \\
\hline
\end{tabular}

Comparison of the two techniques through post-operative echocardiogram. TTE, transthoracic echocardiography; MVA, mitral valve area; MVPG, mean mitral valve pressure gradient; $T R$, triangular resection; FR, folding repair. ventricle (4). In this study, we investigated the difference between the TR and FR techniques in patients with PML lesion and determined whether similar results could be attained with the artificial chordae technique. The use of artificial chordae is a promising technique and has become popular in the repair of the posterior leaflet. However, Seeburger et al. reported that the chordae replacement technique leads to significantly longer operative times, duration of $\mathrm{CPB}$, and aortic cross-clamp times than the leaflet resection technique (5). Yet, our study suggested that there was little difference in the operative time, CPB time, and length of ICU stay between the two techniques. The FR technique makes it easy to remove and reposition when the results are unsatisfactory, allowing for a shorter operative time compared with chordae replacements. This would be advantageous for MICS operation and surgical training purposes.

The degree of residual MR regression to a less than mild level was similar in both groups (Figures 3,4). The risk factors for the late recurrence of moderate or severe MR include fibroelastic etiology, mitral calcification, posterior leaflet plication, non-use of prosthetic ring annuloplasty, and significant pulmonary hypertension (10). Two recurrent cases from the TR group showed degenerative MS changes and had healed IE. Therefore, MR regression within our patients was not associated with previously identified risk factors. Nishida et al. reported that the cause of MV repair failure was valve-related in $71 \%$ of patients, $9 \%$ of which were IE patients. Furthermore, MV re-repair was more common in leaflet suture dehiscence, ring detachment, and neochordae complication procedures (11).

The MVA was significantly larger in the FR group. The
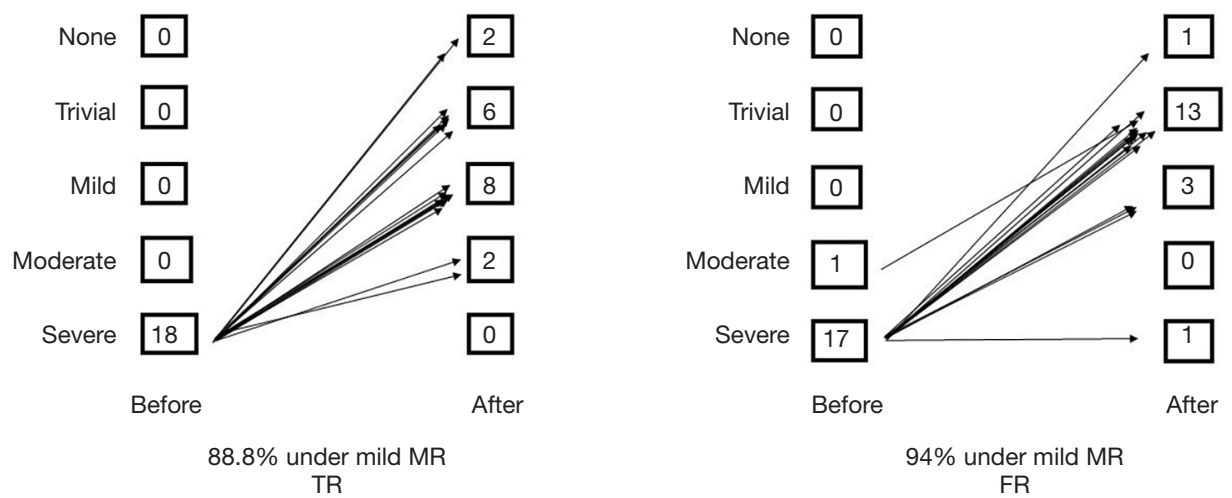

Figure $3 \mathrm{MR}$ assessment after the operation. Mitral regurgitation (MR) regression rate under mild MR in the triangular suture resection (TR) and folding repair (FR) groups. 


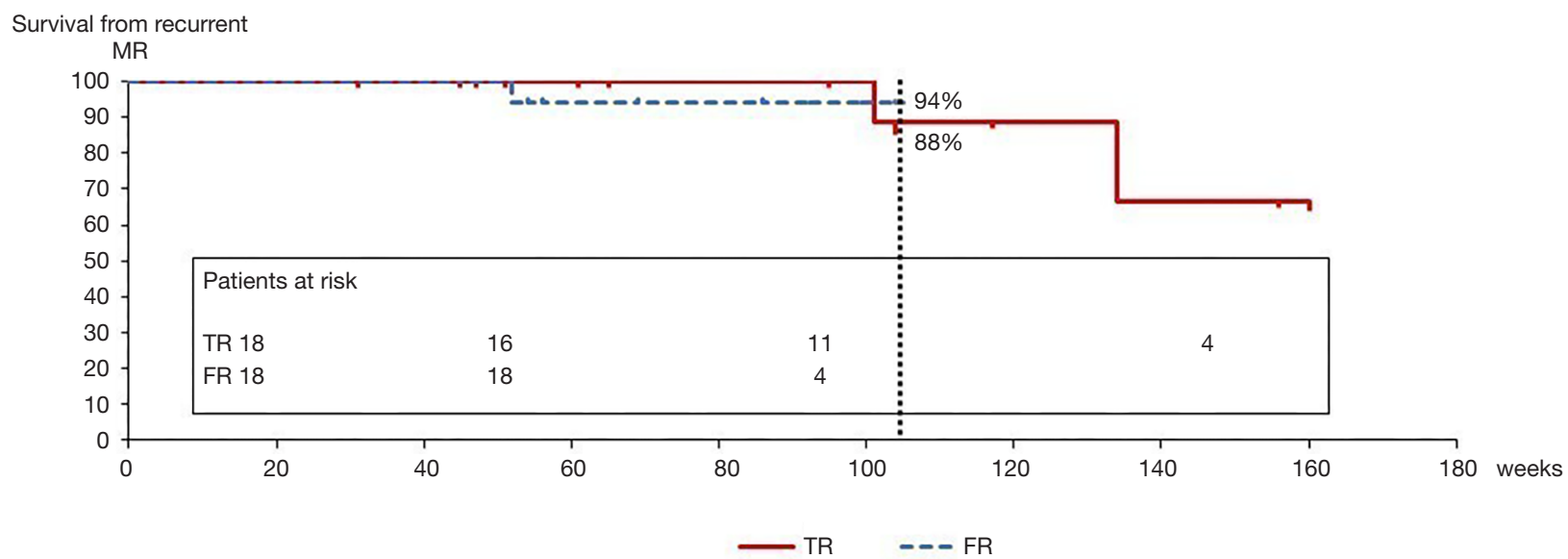

Figure 4 Kaplan-Meier analysis of the short-term outcomes according to recurrent MR) (< mild MR). The reasons for recurrence were degenerative stenotic change [mitral stenosis (MS), $n=2$ ] in the triangular suture resection (TR) group and chordae rupture in the folding repair $(\mathrm{FR})$ group $(\mathrm{n}=1) . \mathrm{I}$

FR group had a lower pressure gradient (PG) than the TR group $(2.8 \pm 0.4$ vs. $3.8 \pm 0.4 \mathrm{mmHg}, \mathrm{P}=0.09)$, but this difference was not significant. However, a larger MVA may provide a lesser PG (12). Similarly, Seeburger et al. reported that after a leaflet-preservation approach with the use of artificial chordae, the mitral orifice area was larger and the mean transvalvular pressure gradients were lower (5). Jahren et al. reported that neochordoplasty, with a ring annuloplasty, resulted in significantly lower transvalvular pressure gradients than leaflet resection in an ex vivo porcine model (13). FR preserved the valve leaflet and subvalvular components as artificial chordae, which led to similar results. Regarding the size of the implanted mitral annuloplasty rings, the FR group allowed for implantation of significantly larger rings. In this small series, there was no clinical difference between the groups but why this varied between the groups. One of the possible reasons for the small ring size in the TR group, despite the fact that the ring size was formally selected based on the size of the anterior leaflet surface area, was that the surgeon may have had a bias to select a smaller ring size in order to gain more coaptation area because of the resection of the posterior leaflet area.

The FR technique is relatively simple and stressfree compared with "resection and suture" or "chordaetransplantation". In cases with deficient valve repair, it is possible to re-initiate FR procedures. The FR technique repairs prolapsed leaflets through insertion into the left ventricle, allowing easy fine-tuning with a pilot suture and repositioning, if unsatisfactory results are obtained. If the
FR technique produces successful results, similar or better than those of well-established techniques, it may be useful, allowing minimally invasive operations, and may also provide an opportunity for less-experienced surgeons to conduct valve repair surgeries (6).

In the TR group, LV function was enhanced after the operation, especially the EF, although this was not significantly different from the FR group $(60.5 \% \pm 4.3 \%$ vs. $56 \% \pm 8.7 \%, \mathrm{P}=0.08)$. In general, $\mathrm{PML}$ resection leads to loss of native valve surface area, altered native valve anatomy, and impairment of physiological function. Thus, the PML is usually stiff, with limited mobility, and with limited coaptation surface area along the anterior leaflet (2). As conceptualized by Carpentier, the goal of mitral valve repair is to restore a good coaptation surface to ensure satisfactory function of the mitral valve (1). The main goal of the RRR approach is to correct the prolapse without leaflet resection (4). Nonresectional dynamic repair techniques are designed to restore normal mitral valve, left ventricular outflow tract, and aortic-mitral coupling function (14).

Through echocardiographic calculations, Imasaka et al. indicated that patients undergoing chordae replacement have superior postoperative $L V$ performance than those undergoing leaflet resection (15). Other groups have suggested that non-resection-based strategies preserve leaflet tissue and restore good leaflet coaptation and mobility $(3,7)$. However, our results showed no significant difference between the TR and FR groups. A meta-analysis of 16 papers by Tourmousoglou et al. showed no difference 
in outcomes between preservation and resection, but it showed that the differences tended to be determined by the experience and knowledge of the surgeon (16).

Although the tendency to avoid resection to treat posterior leaflet prolapse has become more popular, the results of our autopsy cases support Dreyfus' alternative approach based on the "respect when you can, resect when you should" concept (17).

\section{Limitations}

This study utilized a retrospective, single-institution analysis, which may limit its generalizability. The sample size was relatively small; therefore, the results might be biased. The operative selection of either FR or TR was based on the surgeon's preference. Furthermore, the follow-up period was limited, as only the early outcomes and short-term MR recurrence rates were evaluated. A larger patient group and longer follow-up period may be necessary to assess the efficacy and durability of these techniques.

\section{Conclusions}

The TR and FR techniques showed comparable surgical outcomes and durability. However, FR has a benefit in terms of the MVA and pressure gradients, which is expected for an artificial chordae technique and which is relatively simple and may be beneficial for repairing any mitral valve regression and for surgical training purposes.

\section{Acknowledgments}

Funding: None.

\section{Footnote}

Reporting Checklist: The authors have completed the STROBE reporting checklist. Available at http://dx.doi. org/10.21037/cdt-20-760

Data Sharing Statement: Available at http://dx.doi. org/10.21037/cdt-20-760

Conflicts of Interest: All authors have completed the ICMJE uniform disclosure form (available at http://dx.doi. org/10.21037/cdt-20-760). The authors have no conflicts of interest to declare.
Ethical Statement: The authors are accountable for all aspects of the work in ensuring that questions related to the accuracy or integrity of any part of the work are appropriately investigated and resolved. This study conformed to the provisions of the Declaration of Helsinki (as revised in 2013). This study was approved by the institutional review board at the Saitama Cardiovascular and Respiratory Center (No. 2020004). Written informed consent was obtained from the patient for using the data for academic publication purposes. The study was registered with the University Hospital Medical Information Network (study ID: UMIN000039041).

Open Access Statement: This is an Open Access article distributed in accordance with the Creative Commons Attribution-NonCommercial-NoDerivs 4.0 International License (CC BY-NC-ND 4.0), which permits the noncommercial replication and distribution of the article with the strict proviso that no changes or edits are made and the original work is properly cited (including links to both the formal publication through the relevant DOI and the license). See: https://creativecommons.org/licenses/by-nc-nd/4.0/.

\section{References}

1. Carpentier A. Cardiac valve surgery-The "French correction”. J Thorac Cardiovasc Surg 1983;86:323-37.

2. Dreyfus GD, Corbi P, Rubin S, et al. Posterior leaflet preservation in mitral valve prolapse: A new approach to mitral repair. J Heart Valve Dis 2006;15:528-30.

3. Padala M, Powell SN, Croft LR, et al. Mitral valve hemodynamics after repair of acute posterior leaflet prolapse: Quadrangular resection versus triangular resection versus neochordoplasty. J Thorac Cardiovasc Surg 2009;138:309-15.

4. Perier P, Hohenberger W, Lakew F, et al. Toward a new paradigm for the reconstruction of posterior leaflet prolapse: Midterm results of the "respect rather than resect” approach. Ann Thorac Surg 2008;86:718-25.

5. Seeburger J, Falk V, Borger MA, et al. Chordae replacement versus resection for repair of isolated posterior mitral leaflet prolapse: À ègalité. Ann Thorac Surg 2009;87:1715-20.

6. Tsukui H, Umehara N, Saito H, et al. Early outcome of folding mitral valve repair technique without resection for mitral valve prolapse in 60 patients. J Thorac Cardiovasc Surg 2013;145:104-8.

7. Salvador L, Mirone S, Bianchini R, et al. A 20-year 
experience with mitral valve repair with artificial chordae in 608 patients. J Thorac Cardiovasc Surg 2008; 135:1280-7.

8. Gazoni LM, Fedoruk LM, Kern JA, et al. A simplified approach to degenerative disease: Triangular resections of the mitral valve. Ann Thorac Surg 2007;83:1658-64.

9. Mohty D, Orszulak TA, Schaff HV, et al. Very long-term survival and durability of mitral valve repair for mitral valve prolapse. Circulation 2001;104:I1-7.

10. Correia PM, Coutinho GF, Branco C, et al. Surgical treatment of posterior mitral valve prolapse: Towards 100\% repair. J Heart Valve Dis 2015;24:752-9.

11. Nishida H, Fukui T, Kasegawa H, et al. Causes of repair failure for degenerative mitral valve disease and reoperation outcomes. Eur J Cardiothorac Surg 2018;53:1244-50.

12. Murashita T, Greason KL, Suri RM, et al. Mitral valve gradient after valve repair of degenerative regurgitation with restrictive annuloplasty. J Thorac Cardiovasc Surg 2016;151:106-9.

13. Jahren SE, Hurni S, Heinisch PP, et al. Transvalvular pressure gradients for different methods of mitral valve repair: Only neochordoplasty achieves native valve gradients. Interact Cardiovasc Thorac Surg 2018;26:248-55.

14. Lawrie GM, Zoghbi W, Little S, et al. One hundred percent reparability of degenerative mitral regurgitation: Intermediate-term results of a dynamic engineered approach. Ann Thorac Surg 2016;101:576-83.

15. Imasaka K, Tayama E, Tomita Y. Left ventricular performance early after repair for posterior mitral leaflet prolapse: Chordal replacement versus leaflet resection. J Thorac Cardiovasc Surg 2015;150:538-45.

16. Tourmousoglou C, Lalos S, Dougenis D. Mitral valve repair of isolated posterior leaflet prolapse: Resect or respect? Interact Cardiovasc Thorac Surg 2014;19:1027-35.

17. Dreyfus GD, Dulguerov F, Marcacci C, et al. "Respect when you can, resect when you should": A realistic approach to posterior leaflet mitral valve repair. J Thorac Cardiovasc Surg 2018;156:1856-66.e3.
Cite this article as: Nakamura K, Orii K, Abe T, Haida H, Hashimoto K, Kunihara T. Triangular resection versus folding repair for simple posterior mitral leaflet lesions: case-control study. Cardiovasc Diagn Ther 2020;10(6):1839-1846. doi: 10.21037/cdt-20-760 


\section{Supplementary}
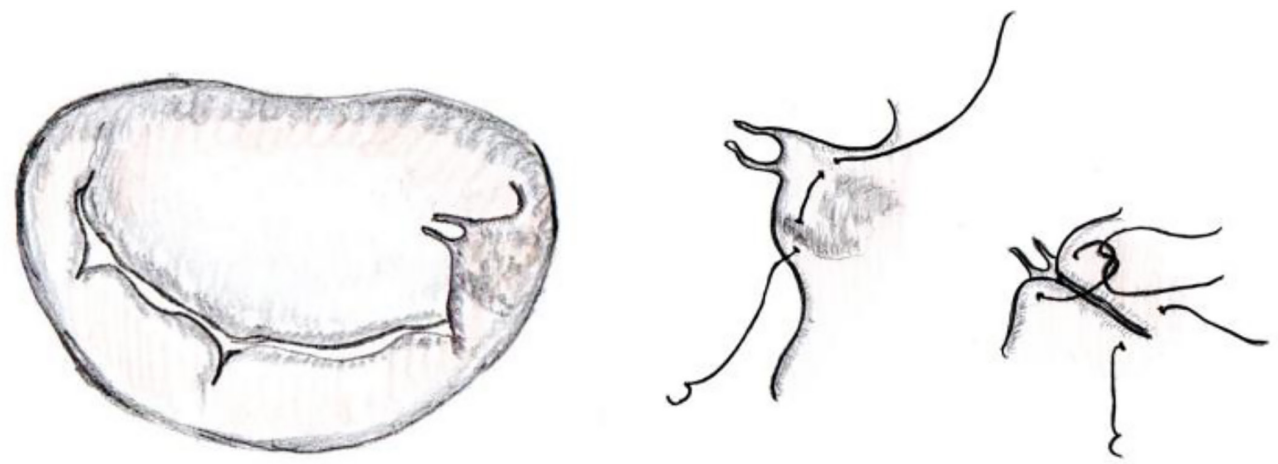

Figure S1 Folding repair. A temporary suture was placed, followed by an additional saline water test. Additional interrupted sutures were placed toward the annulus. 